\title{
Biomass-Derived Bilayer Solar Evaporator with Enhanced Energy Utilization for High-Efficiency Water Generation
}

Fang Yu, Zhenzhen Guo, You Xu, Zihe Chen, Muhammad Sultan Irshad, Jingwen Qian, Tao Mei, Xianbao Wang*

Hubei Collaborative Innovation Center for Advanced Organic Chemical Materials, Ministry-of-Education Key Laboratory for the Green Preparation and Application of Functional Materials, Hubei Key Laboratory of Polymer Materials (Hubei University), School of Materials Science and Engineering, Hubei University, Wuhan 430062, P.R. China.

Figure S1-20

* Corresponding author. Tel.: +86-2788661729; Fax: +86-2788661729.

E-mail: wangxb68@aliyun.com (X.B. Wang) 


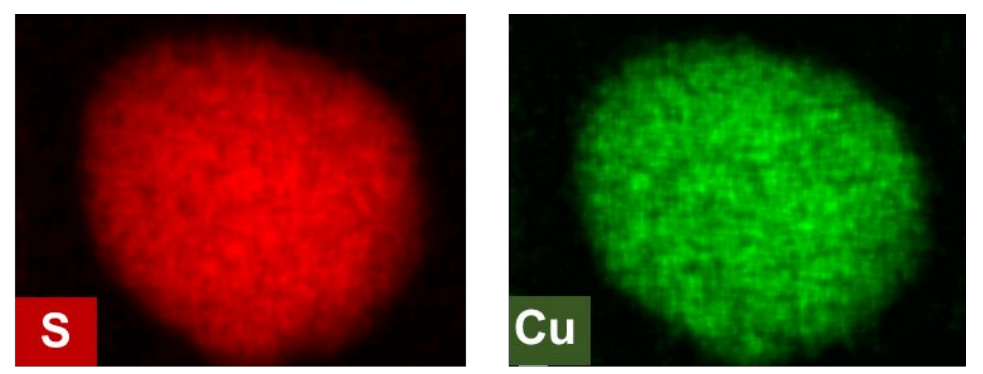

Figure S1. Elemental mapping images of the CuS-PVP4 superstructure.

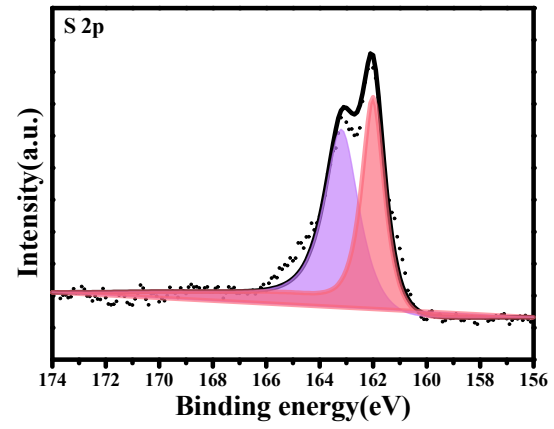

Figure S2. High-resolution XPS spectra of CuS-PVP4 of S 2p. 


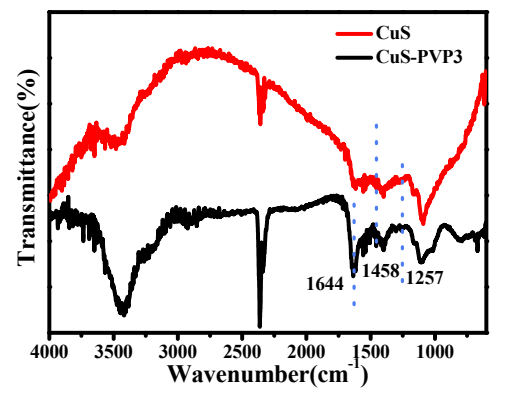

Figure S3. FT-IR spectra of the CuS and CuS-PVP3.

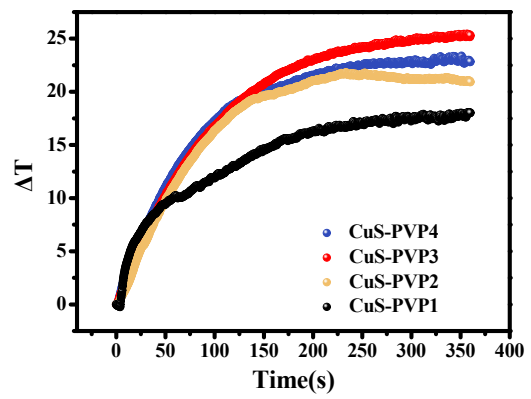

Figure S4. Photothermal performance of CuS-PVP-based aqueous dispersion (200 $\mu \mathrm{g}$ $\left.\mathrm{mL}^{-1}\right)$ under Xe lamp irradiation $\left(2 \mathrm{~kW} \mathrm{~m}^{-2}\right)$ 


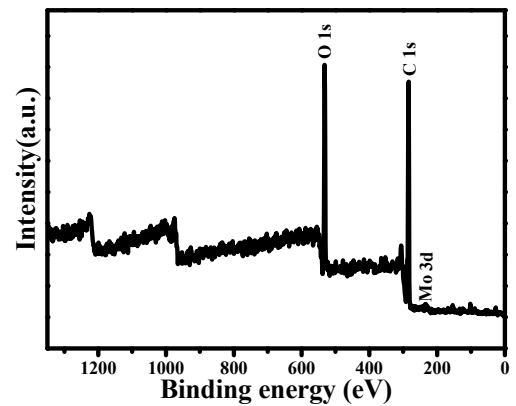

Figure S5. XPS survey spectrum of MoCC.

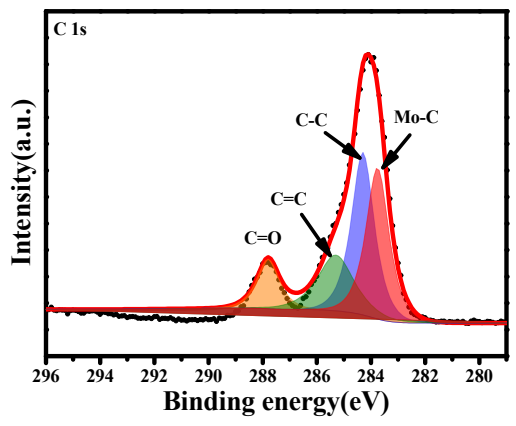

Figure S6. High-resolution XPS spectra of MoCC of C 1s. 


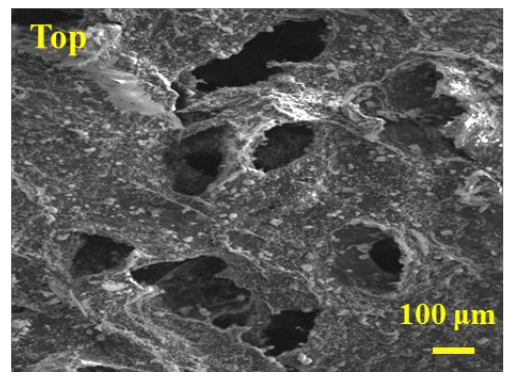

Figure S7. SEM image of the surface of CuSAA/MoCCFA.

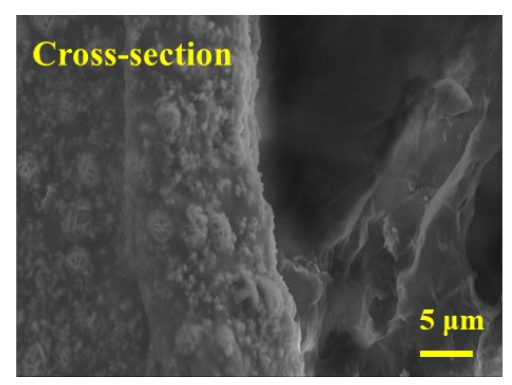

Figure S8. SEM image of the cross-sectional view of CuSAA/MoCCFA. 

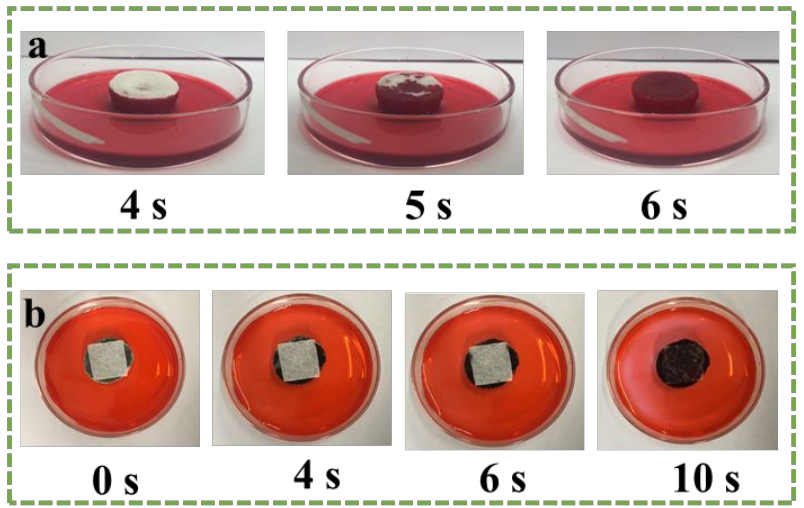

Figure S9. Digital images showing hydrophilic property of pure cellulose aerogel(a) and CuSAA/MoCCFA(b), using the red ink to highlight the water absorption.

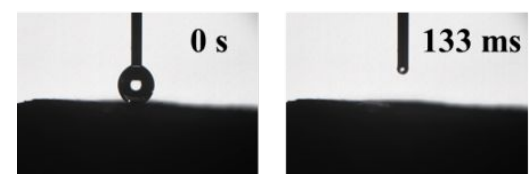

Figure S10. The measurement of water contact angle for CuSAA/MoCCFA. 


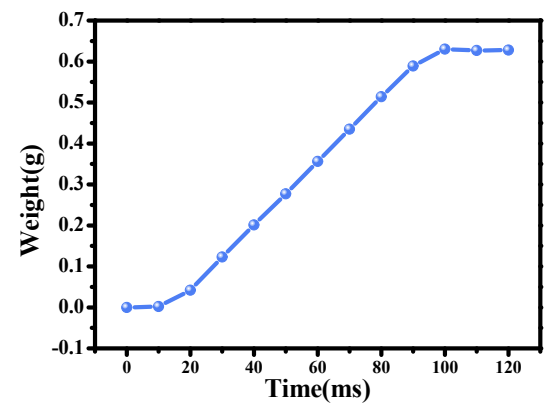

Figure S11. Water adsorption curves of CuSAA/MoCCFA over time under $50{ }^{\circ} \mathrm{C}$.

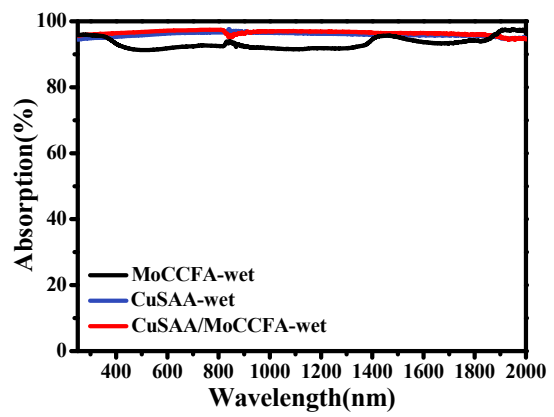

Figure S12. UV-vis-NIR absorption of CuSAA/CFA, MoCCFA and CuSAA/MoCCFA in their wet conditions. 


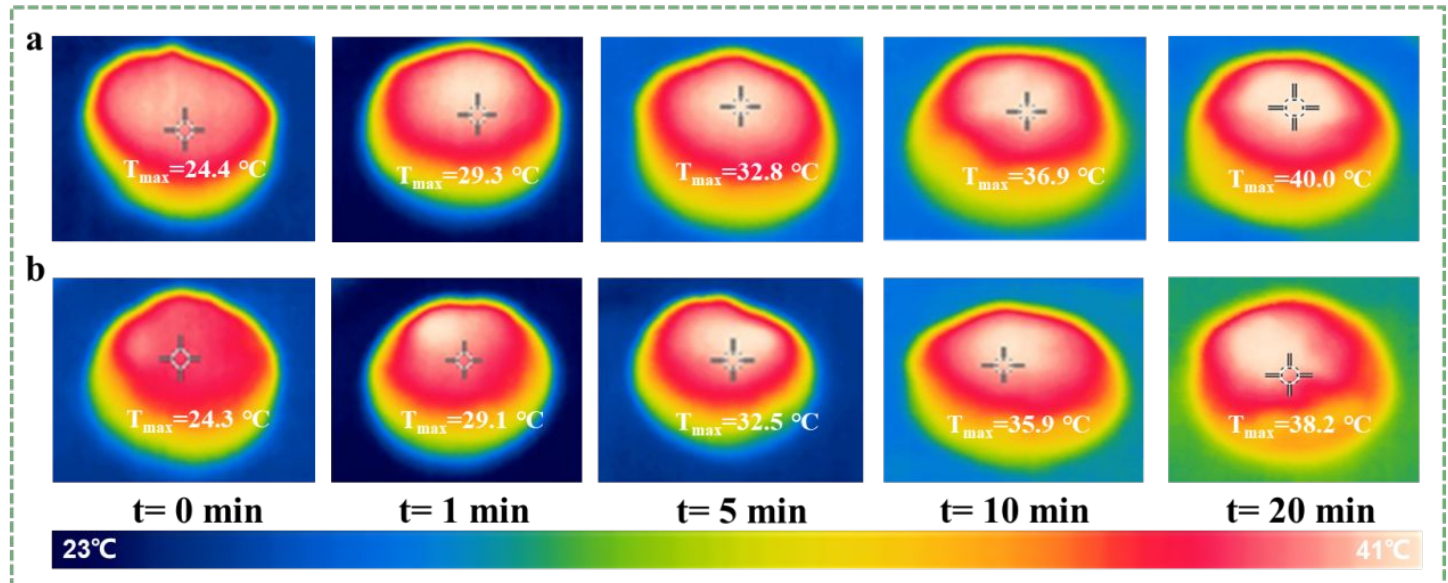

Figure S13. Infrared images of MoCCFA (a) and CuSAA (b) under one sun illumination.

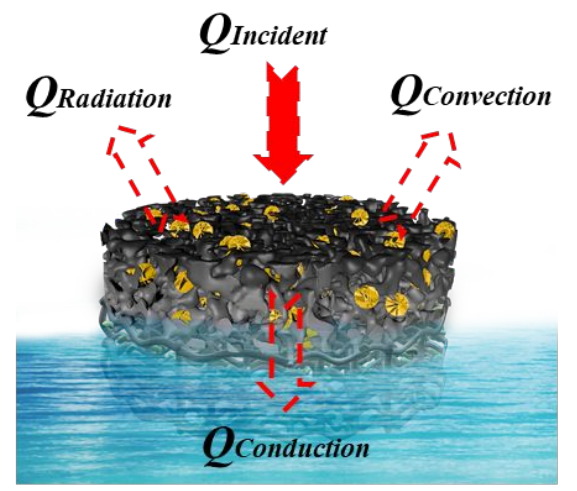

Figure S14. Thermal balance and heat transfer diagram of the solar evaporation device under solar illumination. 

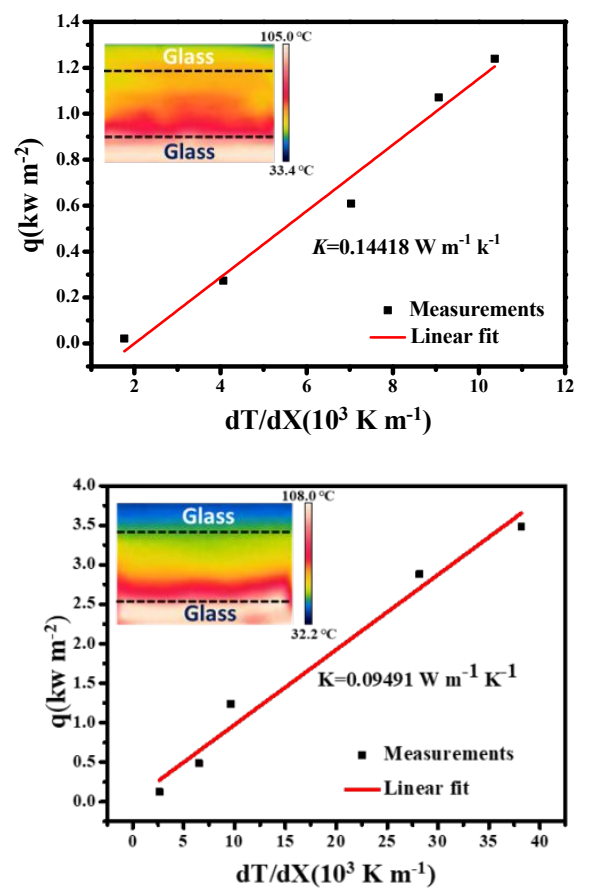

Figure S15. The thermal conductivity of CuSAA/MoCCFA at dry and wet state in air conduction was measured by the IR camera method. The inset is the representative picture of temperature gradient along the thickness of CuSAA/MoCCFA.

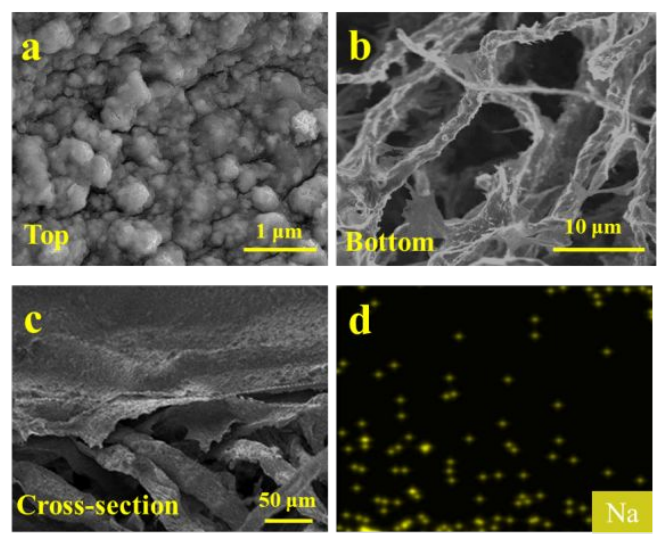

Figure S16. (a-c) The SEM images of CuSAA/MoCCFA, and the elemental mapping of $\mathrm{Na}$ in the porous MoCCFA layer (d) after 15 cycles of solar desalination in the $20 \mathrm{wt} \% \mathrm{NaCl}$ solution. 


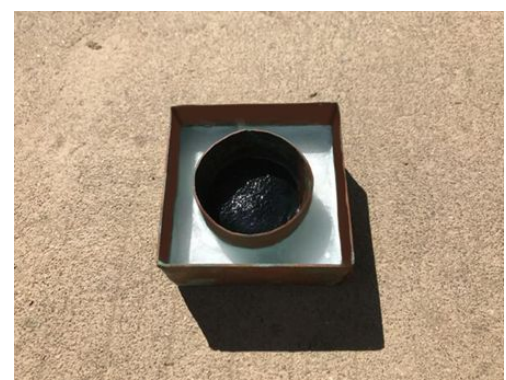

Figure S17. Digital photograph of hybrid evaporation system composing of CuSAA/MoCCFA and low molten salt.

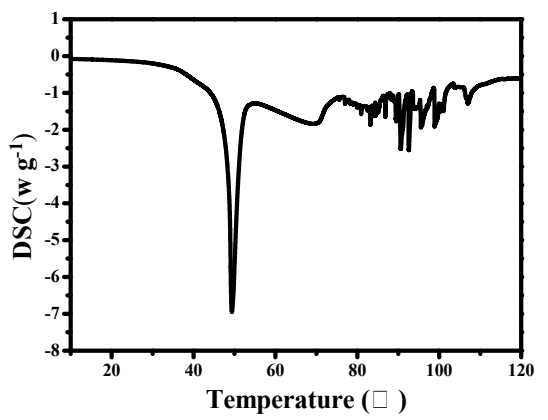

Figure S18. DSC curves of the as-prepared PCMs. 


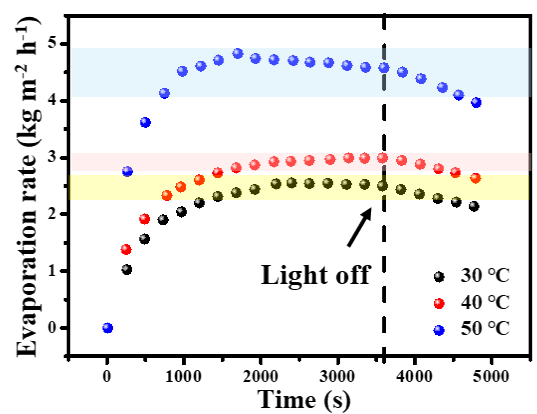

Figure S19. Solar evaporation rates of water of CuSAA/MoCCFA with different simulated temperatures.

\section{The energy consumption calculation during solar-driven water evaporation was conducted.}

In order to analysis the energetic balance between solar input and the energy loss to the air includes heat conduction, heat convection and heat radiative, the thermodynamic equilibria were verified by stable surface temperature under sunlight illumination according to the thermal transportation theories. The main pathway of heat loss from the absorber under irradiation includes three portions: conductive heat loss to bulk water $\left(Q_{\text {Conduction }}\right)$, convective $\left(Q_{\text {Convection }}\right)$ and radiative $\left(Q_{\text {Radiation }}\right)$ heat loss to the surroundings.

\subsection{Light absorbing loss}

Due to the discrepancy in solar absorption properties of evaporators, light absorbing loss existed in our experiments. For example, the light absorbing percentage $(\alpha)$ over the solar radiation spectra of wet CuSAA/MoCCFA is almost $97 \%$. Therefore, the absorbed energy of evaporator is nearly $970 \mathrm{~W} \mathrm{~m}^{-2}$.

\subsection{Conductive heat loss}

The conductive heat loss to bulk water $\left(Q_{\text {Conduction }}\right)$ can be calculated as:

$Q_{\text {Conduction }}=A k\left(T_{1}-T_{2}\right) / \Delta l$

where $A$ is the cross-sectional area $\left(5 \times 2 \mathrm{~cm}^{2}\right), k$ denotes the thermal conductivity of bulk water $\left(0.599 \mathrm{~W} \mathrm{~m}^{-1} \mathrm{~K}^{-1}\right)$. The two embedded thermocouples were applied to monitor the water temperature, and the distance between the thermocouples $(\Delta l)$ is 5 $\mathrm{mm}$. The temperature evolutions were shown in Figure S20. The average temperature at Point 1 over one hour is about $27.10^{\circ} \mathrm{C}$. The average temperature at Point 2 over one hour is about $26.06^{\circ} \mathrm{C}$. Consequently, the $Q_{\text {Conduction }}$ is estimated as $12.46 \mathrm{~W} \mathrm{~m}^{-2}$, corresponding to $1.25 \%$.

\subsection{Convective heat loss}

The convective heat loss $\left(Q_{\text {Convection }}\right)$ to surroundings can be calculated as:

$Q_{\text {Convection }}=h\left(T_{s}-T_{v}\right)(\mathrm{S} 7)$

where $h$ denotes convection heat transfer coefficient (assumed to be $10 \mathrm{~W} \mathrm{~m}^{-2} \mathrm{~K}^{-1}$ ), 
and Ts is the surface temperature of the solar evaporator $\left(41.4{ }^{\circ} \mathrm{C}\right)$. Since the surface is surrounded by hot vapor, the surrounding temperature can take the vapor temperature $\left(40.1{ }^{\circ} \mathrm{C}\right)$. Consequently, the $Q_{\text {Conduction }}$ is estimated as $13 \mathrm{~W} \mathrm{~m}^{-2}$, corresponding $1.34 \%$.

\subsection{Radiative heat loss}

The radiative heat loss $\left(Q_{\text {Radiation }}\right)$ to surroundings can be calculated as:

$Q_{\text {Radiation }}=\varepsilon \sigma\left(T_{s}^{4}-T_{\infty}{ }^{4}\right)(\mathrm{S} 8)$

where $\varepsilon$ denotes the emission of the absorber (assumed to be 0.93), $\sigma$ is the Stefan-Boltzmann constant $\left(5.669 \times 10^{-8} \mathrm{~W} \mathrm{~m}^{-2} \mathrm{~K}^{-4}\right), T_{\infty}$ is the temperature of the adjacent environment. Since the surface is surrounded by hot vapor which is semitransparent to thermal radiation, the radiative heat losses may be within the range that is delimited by the maximum and minimum values calculated by using $T_{\infty}=T_{a}$ (ambient temperature of $26.0{ }^{\circ} \mathrm{C}$ ) and $T_{\infty}=T_{v}$ (vapor temperature of $41.0{ }^{\circ} \mathrm{C}$ ), respectively.

When $\mathrm{T}_{\infty}=\mathrm{T}_{\mathrm{a}}=26.0{ }^{\circ} \mathrm{C}$, the radiative heat loss is estimated as $17.78 \mathrm{~W} \mathrm{~m} \mathrm{~m}^{-2}$, corresponding to $1.83 \%$.

When $T_{\infty}=T_{v}=41.0{ }^{\circ} \mathrm{C}$, the radiative heat loss is estimated as $16.06 \mathrm{~W} \mathrm{~m}^{-2}$, corresponding to $1.66 \%$. Therefore, the radiative heat loss in this work falls in the range of $1.66 \sim 1.89 \%$.

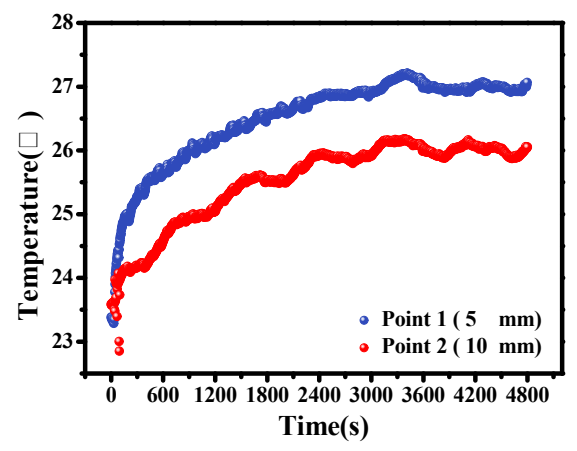

Figure S20. Temperature evolutions underneath the water. The temperatures were monitored by T-type thermocouples. Point 1 and Point 2 were placed $\sim 5 \mathrm{~mm}$ and $\sim 10$ $\mathrm{mm}$ below the bottom surface of CuSAA/MoCCFA, respectively. 